\title{
Prevalence and Distribution of Oral Conditions among Dental out Patients Children Aged 0-13 Years Attending Khanzad Polyclinic Center in Erbil City
}

\author{
Banaz A. Abdullah', Shaheen A. Ahmed', Ali F. Mohammad Alzubaidee ${ }^{3}$ \\ ${ }^{1}$ (KBMS) Kurdistan Board Candidate of Oral and Maxillofacial Medicine, Erbil, Kurdistan Region, Iraq, ${ }^{2}$ Department of Oral and Maxillofacial \\ Surgery, College of Dentistry, Hawler Medical University, Erbil, Kurdistan Region, Iraq, ${ }^{3}$ Consultant at Kurdistan Board of Medical Specialties, \\ Head of Council of Dental Specialties, Erbil, Kurdistan Region, Iraq
}

\section{${ }^{*}$ Corresponding author: \\ Banaz A. Abdulla, (KBMS) Kurdistan Board Candidate of Oral and Maxillofacial Medicine, Erbil, Kurdistan Region, Iraq. \\ E-mail: banazrt@gmail.com}

Received: 11 April 2020

Accepted: 13 July 2020

Published: 30 December 2020

\section{DOI}

10.25156/pti.v10n2y2020.pp81-86

\section{A B S TR A C T}

Prevalence studies on oral conditions affecting children are rare worldwide, and none of them was conducted in in Erbil/Kurdistan region/Iraq. The aims in the this study were to determine the prevalence and distribution of oral conditions which include both lesions and normal variations in group of patients referred to a main, public specialized poly clinics dental center. A prospective cross-sectional study was performed, the including data collected were age, gender, and site of a condition. The examination of the patients was performed under standardized conditions; using artificial light, disposable gloves, retractors, and mirror with sterile gauze to hold tip of the tongue. Statistical analysis was performed using Statistical Package for the Social Sciences (SPSS) version 22; Fisher's exact test; and Chisquare test. A sample of 520 children, aged range (0-13) years included in this study, $265(51.0 \%)$ were males and $255(49.0 \%)$ were females, divided into three age groups; $100(19.2 \%)$ were $<5$ years, $274(52.7 \%)$ were $5-9$ years, and $146(28.1 \%)$ were $10-13$ years. The prevalence of oral conditions was significantly higher in $5-9$ years $(52.7 \%)$. The most commonly clinically diagnosed oral conditions were fissure tongue $(13.1 \%)$, followed by gingivitis $(9.0 \%)$ and dentoalveolar sinus $(8.3 \%)$. As a first study in this age group in Erbil/Kurdistan region/Iraq, oral conditions are relatively common in the sample examined; dentist and dental practitioner should be aware for their recognition and referral for management.

Keywords: Oral mucosal lesions; Normal variations; Pediatric population; Cross-sectional study; Government dental center

\section{INTRODUCTION}

Oral mucosal abnormality whether lesions or normal variations among pediatrics show a spectrum which distinguished from that shown in adults, by being relating to growth and development of the child, such as conditions more predominate in the such age group, such as vascular tumors and some types of lymphoma (Dhanuthai et al., 2007 and Puangwan et al., 2015). That is why signs and symptoms are different between the two mentioned age groups. Due to continuous emotional and biological changes in children, various types of alterations and lesions in the orofacial region can take place (Sandeepa et al., 2013). The awareness of the general dental practitioner and the pediatric dentist of the common oral conditions, their etiology, and treatment will facilitate proper diagnosis and their management or even prevention of these conditions.

Despite World Health Organization recommendations (Petersen et al., 2005), only a few studies were performed to investigate the prevalence of oral conditions among children. The precise prevalence of oral conditions in healthy children is controversial due to a lack of standardized methods, different diagnostic criteria, and the description of very few conditions in each survey (Bessa et al., 2004, Aldred et al., 2008 and Crespo et al., 2005). The aims of the current study were to detect the prevalence and distribution of oral conditions among children $0-13$ years old that are visiting Specialized Khanzad Polyclinic Teaching Center in Erbil/Kurdistan region/Iraq.

\section{PATIENTS AND METHOD}

\section{Selection of a Sample}

A prospective cross-sectional study was designed, including 520 pediatric patients, ( $0-13$ included) years of age, of different nationality (mostly Kurdish), using clinical charts from the Department of Diagnosis of Specialized Khanzad Teaching Center/Erbil. All patients were examined for the 
presence of oral conditions. Since some papers divided the oral cavity into parts, the decision in this work was made to divide it into; tongue conditions (TC), lip conditions (LC), and conditions affecting other oral sites (OC). The data were collected from October 2019 to January 2020 including socio-demographic variables of the patients; age, gender, site of conditions, and date of examination. The study was approved by the research ethical committee of the Kurdistan Board of Medical Specialties. Parents or caregivers of all patients were interviewed regarding chief symptoms and history of current illness, medical, drug, and dental history. Their awareness of the present condition had been reported. Heterogeneous sample with or without systemic disease, and use of medications was included in this study. Handicapped patients and dental caries were excluded from the study.

\section{Examination and Data Recording}

Systematic extra- and intra-oral examination was performed by the first author, after taking verbal consent from their parents, with the implementation of the standardized infection control measures in accordance with Occupational Safety and Health Act guidelines (Boyce and Mull, 2008). Oral examinations were performed under standardized conditions; using artificial light, disposable gloves, retractors, and mirror, with sterile gauze to hold tip of the tongue. Medical and drug histories had been noted according to the information given by patients and parents. After finishing the case sheet, all patients were referred to a specialized clinic for managements. Mentioned in all 4 Figures 1-5.

\section{Statistical Analysis}

Data were analyzed using the Statistical Package for the Social Sciences (SPSS, version 22). Microsoft Excel was used to draw the charts. Means and standard deviation (SDs) were used to summarize the numerical variables, and frequencies and proportions were used to describe the categorical variables. Chi-square test of association was used to compare

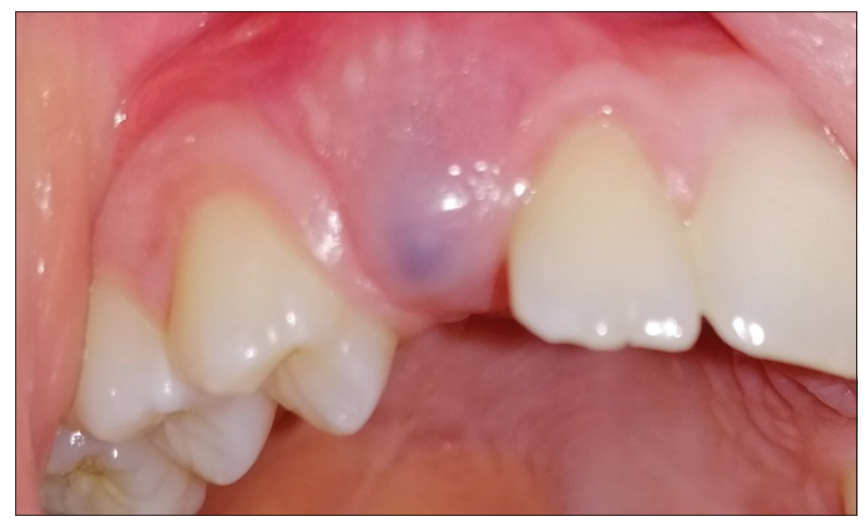

Figure 1: Eruption cyst presented as a bluish swelling at area of unerupted maxillary right permanent canine proportions. Fisher's exact test was used when the expected count of more than $20 \%$ of the cells of the table was $<5$. $P \leq 0.05$ was considered statistically significant.

\section{RESULTS}

The total number of patients was 520 . Their mean age \pm $\mathrm{SD}$ was $7.47 \pm 3.02$ years, ranging from 0.0054 years $(2$ days) until 13 years which included. The median was 7.5 years $21(4 \%)$ children only had history of systemic diseases with no effect on the oral conditions. The most common one was Glucose 6 Phosphate Dehydrogenase deficiency and only $7(1.3 \%)$ children were taking drugs. Table 1 shows more than half $(52.7 \%)$ of the sample aged $5-9$ years, $51 \%$ were males and $66.2 \%$ were students.

It is found that more than half $(54.4 \%)$ of the patients had oral conditions, in which $32.7 \%$ were OC, $26.3 \%$

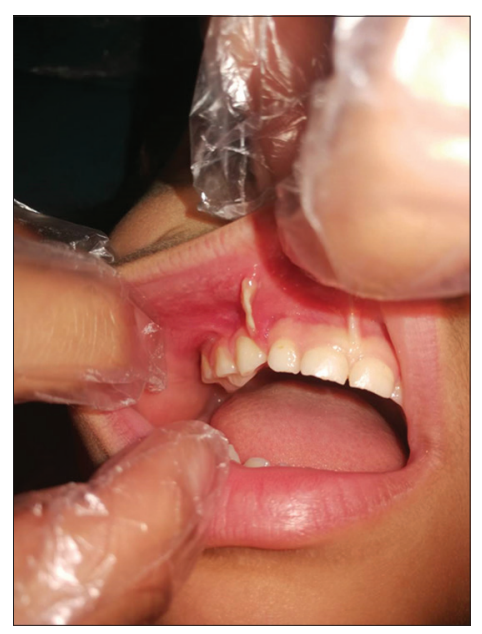

Figure 2: Intraoral picture of 5 years old female showing fibrotic tissue at the right maxilla extending from alveolar ridge between $B$ and $C$ to labial mucosa. representing accessory oral frenum

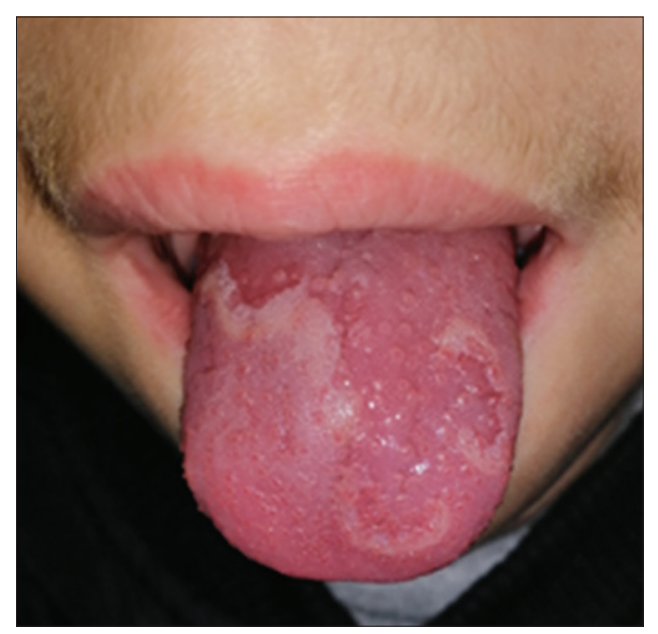

Figure 3: Geographic tongue presented as multiple red areas surrounded by white boarder on the dorsal surface of the tongue 


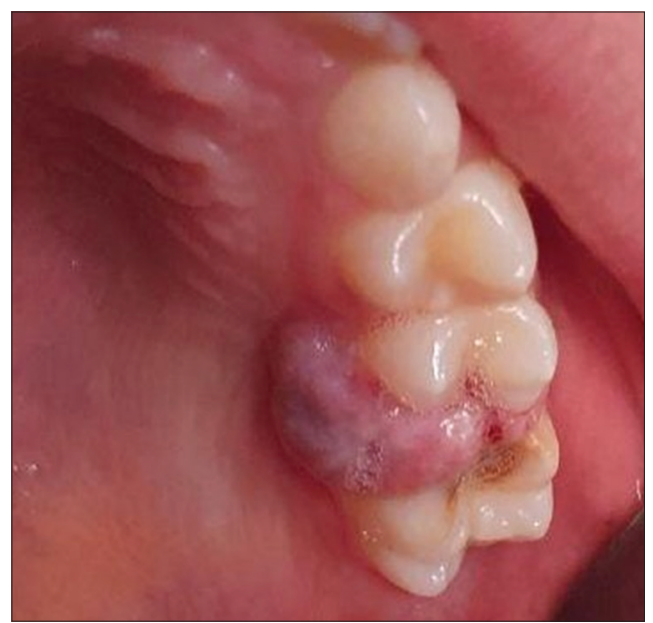

Figure 4: A lobulated bluish pink swelling in the interdental area between upper left $2^{\text {nd }}$ premolar and $1^{\text {st }}$ permanent molar

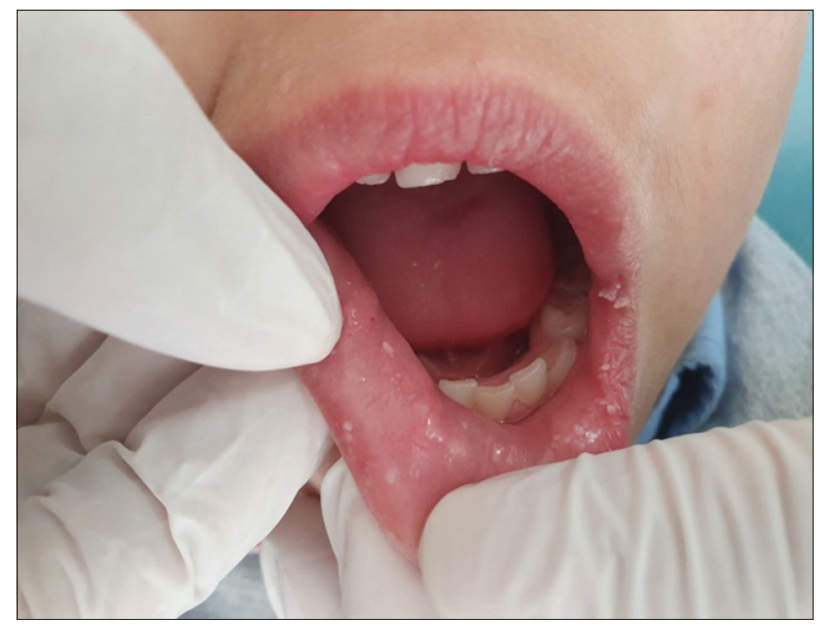

Figure 5: Showing multiple small white warts like lesion at the left commissure and bilateral lower labial mucosa

Table 1: Demographic characteristics of the studied sample

\begin{tabular}{lc}
\hline Sample characteristics & $\boldsymbol{n}(\%)$ \\
\hline Age (years) & \\
$<5$ & $100(19.2)$ \\
$5-9$ & $274(52.7)$ \\
$10-13$ & $146(28.1)$ \\
Mean \pm SD & $7.47 \pm 3.02$ \\
Gender & \\
Male & $265(51.0)$ \\
Female & $255(49.0)$ \\
Occupation & \\
Student & $344(66.2)$ \\
Left the school & $12(2.3)$ \\
Pre-school & $164(31.5)$ \\
Total & $520(100.0)$ \\
\hline
\end{tabular}

SD: Standard deviation

were TC, and $4.4 \%$ were LC, and more than half $(56 \%)$ of the sample were aware of their conditions either by themselves or their parents, but it was clear that the majority $(82.6 \%)$ of patients with LC were aware of it, compared with $63.9 \%$ for OC and $41.6 \%$ for TC, the more the age, the more the prevalence of TC $(P<0.001)$ and $\mathrm{OC}(P=0.004)$. No significant association was detected between age and LC $(P=0.233)$. No significant association was found between gender and the prevalence of the following conditions: TC $(P=0.526)$, LC $(P=0.758)$, and OC $(P=0.760)$. The prevalence of TC was $36.1 \%$ among those who were aware of their condition while it was significantly high (64.5\%) among those who were unaware of their condition $(P<0.001)$. The reverse pattern was observed regarding the LC and OC, it was evident that the prevalence was higher among those who were aware about their condition compared with those who were unaware $(P=0.007$ and $P=0.001)$, respectively. The prevalence rates of the oral conditions among 520 pediatric patients are shown in Table 2 which is combined table of the three oral sites.

Tables 3 and 4 show the main three oral conditions for each site with no significant difference neither by gender nor age, except for the melanotic stain, which was significantly higher among males $(10.2 \%)$ than among females $(5.5 \%)$ $(P=0.047)$ and increased significantly with age $(P<0.001)$. The prevalence of gingivitis was high $(7.8 \%)$ among those aged 10-13 compared with 7.3 and $1 \%$ among those aged (5-9 and $<5)$ years, respectively, $(P<0.001)$. the results showed that the youngest patient with a mouth condition was aged 0.0054 years ( 2 days old), and the oldest was 13 years old. The mean age was $8.18 \pm 2.87$ years, the median was 8 years.

\section{DISCUSSION}

This study was the first to investigate the prevalence of oral conditions in children attending a public dental center/Specialized Khanzad Polyclinic Teaching Center in Erbil city. Cross-sectional studies are important in estimating the prevalence of OC and in identifying highrisk individual. In this study, the male:female ratio was (1.04:1), slight male predominance had been noticed, agree with studies (Cavalcante et al., 2016, Ha et al., 2014 and Patil et al., 2017), and disagree with Taweevisit et al. (2018) and Ambika et al. (2011) both showed slight female predominance. The prevalence of oral conditions in this study was $54.4 \%$ which was higher than reported in the nearby countries such as Turkey (26.2\%) (Parlak et al., 2006) and Iran (28\%) (Jahanfar et al., 2012), possibly because the sample of the current study was from a dental center and not from general hospital, beside; high number of subjects was examined, but it was lower than a study done in India (Ambika et al., 2011), in which 1003 children 4-14 years were included in the study, the prevalence was $64.11 \%$. The different prevalence in studies may be 
Table 2: Prevalence of oral conditions

\begin{tabular}{lc}
\hline Prevalence & $n^{*}=520, n(\%)$ \\
\hline Fissure tongue & $68(13.1)$ \\
Gingivitis & $47(9.0)$ \\
Dentoalveolar sinus & $43(8.3)$ \\
Coated tongue & $42(8.1)$ \\
Melanotic stains & $41(7.9)$ \\
Geographic tongue & $24(4.6)$ \\
Ankyloglossia & $16(3.1)$ \\
Recurrent aphthae & $15(2.9)$ \\
Traumatic injury including bite & $12(2.4)$ \\
Herpes labials & $9(1.7)$ \\
Hereditary gingival fibromatosis & $9(1.7)$ \\
Herpetic stomatitis & $5(1.0)$ \\
Hyper plastic labial frenum & $5(1.0)$ \\
Angular cheilitis & $5(1.0)$ \\
Exfoliative cheilitis & $5(1.0)$ \\
Pyogenic granuloma & $4(0.8)$ \\
Oral candidiasis & $4(0.8)$ \\
Accessory frenum & $4(0.8)$ \\
Lip scar (two treated cleft lip) & $3(0.6)$ \\
Periodontitis & $2(0.4)$ \\
Cleft palate (untreated) & $2(0.4)$ \\
Glossitis & $2(0.4)$ \\
Mucocele & $1(0.2)$ \\
Eruption cyst & $1(0.2)$ \\
Oral wart & $1(0.2)$ \\
Red patch(floor of the mouth) & $1(0.2)$ \\
White patch(floor of the mouth) & $1(0.2)$ \\
Black hairy tongue & $1(0.2)$ \\
Median rhomboid glossitis & $1(0.2)$ \\
Lip fissure & $1(0.2)$ \\
Unilateral cleft lip (untreated) & $1(0.2)$ \\
Lip eczema & $1(0.2)$ \\
*n lip swelling (infection) & $1(0.2)$ \\
\hline
\end{tabular}

${ }^{*} n$ is a total number

Table 3: Prevalence of the main oral conditions for each site by gender

\begin{tabular}{lccc}
\hline Oral conditions & $\begin{array}{c}\text { Male }(\mathrm{n}=265), \\
n(\%)\end{array}$ & $\begin{array}{c}\text { Female }(n=255), \\
n(\%)\end{array}$ & $P$ \\
\hline (TC) Fissure & $38(14.3)$ & $30(11.8)$ & $0.384^{* *}$ \\
tongue & & & \\
Coated tongue & $21(7.9)$ & $21(8.2)$ & $0.897^{* *}$ \\
Geographic tongue & $13(4.9)$ & $11(4.3)$ & $0.748^{* *}$ \\
(LC) Herpes labials & $6(2.3)$ & $3(1.2)$ & $0.505^{*}$ \\
Angular cheilitis & $3(1.1)$ & $2(0.8)$ & $0.999^{*}$ \\
Exfoliative cheilitis & $1(0.4)$ & $4(1.6)$ & $0.208^{*}$ \\
(OC) Gingivitis & $23(8.7)$ & $24(9.4)$ & $0.771^{* *}$ \\
Dentoalveolar sinus & $21(7.9)$ & $22(8.6)$ & $0.771^{* *}$ \\
Melanotic stains & $27(10.2)$ & $14(5.5)$ & $0.047^{* *}$ \\
\hline
\end{tabular}

*By Fisher's exact test, **By Chi-square test. TC: Tongue conditions, LC: Lip conditions, OC:Other oral site conditions

attributed to variations in the diagnostic criteria, method of lesions detection employed, examiners training, and calibration (Furlanetto et al., 2012). Besides, differences in study design, sample size, period of study, and age-range stratification. The prevalence of conditions in this study
Table 4: Prevalence of the main oral conditions for each site by age

\begin{tabular}{lcccc} 
Oral conditions & \multicolumn{3}{c}{ Age (years) } & $P$ \\
\cline { 2 - 4 } & $\begin{array}{c}<5 \\
(n=100),\end{array}$ & $\begin{array}{c}5-9 \\
n=274),\end{array}$ & $\begin{array}{c}10-13 \\
(n=146),\end{array}$ \\
& $n(\%)$ & $n(\%)$ & $n(\%)$ & \\
\hline (TC)Fissured tongue & $4(4.0)$ & $34(12.4)$ & $30(20.5)$ & $0.001^{* *}$ \\
Coated tongue & $2(2.0)$ & $27(9.9)$ & $13(8.9)$ & $0.043^{* *}$ \\
Geographic tongue & $3(3.0)$ & $12(4.4)$ & $9(6.2)$ & $0.491^{* *}$ \\
(LC) Labial herpes & $1(1.0)$ & $3(1.1)$ & $5(3.4)$ & $0.219^{*}$ \\
Angular cheilitis & $1(1.0)$ & $3(1.1)$ & $1(0.7)$ & $>0.999^{*}$ \\
Exfoliative cheilitis & $0(0.0)$ & $3(1.1)$ & $2(1.4)$ & 0.713 \\
(OC) Gingivitis & $1(1.0)$ & $20(7.3)$ & $26(17.8)$ & $<0.001^{* *}$ \\
Dentoalveolar sinus & $5(5.0)$ & $29(10.6)$ & $9(6.2)$ & $0.123^{* *}$ \\
Melanic stains & $0(0.0)$ & $17(6.2)$ & $24(16.4)$ & $<0.001^{* *}$ \\
\hline
\end{tabular}

*By Fisher's exact test, **BB Chi-square test. TC: Tongue conditions,

LC: Lip conditions, OC: Other oral site conditions

generally increased with age, probably due to the nature of the conditions; also the awareness of the older children about their conditions, this result consistent with Taweevisit et al. (2018) and Bessa et al. (2004), a study in 1211 Brazilian children, the frequency of oral conditions increased with age, in which some of them were associated with habits and systemic problems. In general, OC was reported as most frequent affected site $(32.7 \%)$ in the current study, while in an American (Shulman, 2005) and Jordanian (Hussein et al., 2017) studies, the lip and tongue were the most affected site 30.70 and $35.9 \%$, respectively. This may emphasizes the importance of the careful examination of all parts of the oral cavity. Among 33 oral conditions in this study, we will discuss the only top five; because of their highest prevalence rate; besides, an observable difference in the prevalence between melanotic stain and geographic tongue.

\section{Fissured Tongue (FT)}

The most prevalent oral condition in this study, was fissured tongue, with the prevalence $(13.1 \%)$ which is consistent with Unur et al., (2015), a study among 1041 Turkish children. While other study in Brazil (Crespo et al., 2005), including 2356 children on investigating oral conditions, tongue diseases were found to have the lowest rate. Literature review revealed wide variability in the prevalence of FT among children word wide by geographic location varied from 1.49 to $23 \%$ (Crespo et al., 2005). A study by Yarom et al. (2004), of different ethnic origins, found the higher FT prevalence $(30.5 \%)$ than the reported range. The prevalence $(13.1 \%)$ in this study is within the mentioned range, and much higher than $(0.38 \%)$, reported by Majorana et al. (2010), but lowers than $(26.7 \%)$, that reported by Al-Maweri et al. (2014).

FT in this study is mostly prevalent (20.5\%), among patients aged 10-13 years compared with $(4 \%)$, those aged $<5$ years $(P=0.001)$, this indicates an increase in the rate of FT in advanced ages, similar results were found in studies by Unur et al. (2015) and Yarom et al. (2004). This leads 
to the idea that characteristic features of the condition are being noticeable with development (Crespo et al., 2005).

In the current study, the number of FT coexisting with geographic tongue was found to be $8(1.5 \%)$, as some researchers found a correlation between them (Dos Santos et al., 2004). This study goes with Hussein et al. (2017), higher number among males had been reported, whereas Ambika et al., (2011), showed nearly equivalent result between either sexes, same as Unur et al. (2015).

\section{Gingivitis}

The second most common oral condition (9.0\%), using gingival index (Löe and Silness, 1963) with no gender predilection but increased with age. In a study done in UK (Jones and Franklin, 2006), the prevalence of 9.96\% was reported for all gingival and periodontal pathology, among 439 children, 39 were diagnosed with chronic gingivitis mostly in older male groups. Ambika et al. (2011) was diagnosed gingivitis as the most commonly observed oral condition (56.43\%), in $268(26.72 \%)$ males and 298 $(29.71 \%)$ females.

Many studies have concluded that gingivitis begins in early childhood age, but will rise at puberty (Stephen, 2010). This can be proved in the result of current study as $(17.8 \%)$ prevalence among $10-13$ years in comparison to $1.0 \%$ among age group $<5$ years had been reported. Although hormonal change can play a role, but improper teeth brushing technique and inadequate time or even lack of oral hygiene practice might be considered. Frequency of gingivitis always difficult to determine due to the lack of agreement on measurement criteria. The high percentage of gingivitis in this study demands for more: Dental hygienist specialists, preventive education care programs including schools and regular screening.

\section{Dentoalveolar Sinus (DAS)}

The third most prevalent oral condition, in comparison with Hussein et al. (2017), the percentage is higher 8.3 and $2.3 \%$, respectively, in the age group of $5-9$ years, with no significant gender difference in the current study, in contrast with a significant higher percentage among female in the other. Ambika et al., (2011), found that only (3.2\%) of children had DAS mostly found in age groups $8-10$ years highly observed among males. The high percentage of DAS indicates high carries rate in deciduous teeth that may targeting the future permanent teeth due to lack of motivation of parents, so preventive measure should be enhanced at early ages.

\section{Coated Tongue (CT)}

CT has not considered by some authors neither as disease nor abnormal alteration (Dos Santos et al., 2004). The prevalence in this study was $(8.1 \%)$, mostly found among 5-9 years, with no gender predilection. In comparing to Hussein et al. (2017), among same age group children only $(4 \%)$ had been reported mostly males.

Different studies with different results have been mentioned, such as: Garcia-Pola et al. (2002), among the 6 years old Spanish children, described CT as the most frequently encountered oral condition with the prevalence of $16.0 \%$, and $23.4 \%$ in preschool Brazilian children (Vieira-Andrade et al., 2017), both showed higher results than the current study, while Majorana et al., (2010), reported a lower prevalence $7.23 \%$ in $0-12$ year old Italian children. Since CT might be a sign for poor oral hygiene, this emphasizes for giving instruction in our population on good oral hygiene performance by the dentists, pediatric dentists, dental practitioner, teachers, and from social medias.

\section{Melanotic Pigmentation or Stain (MS)}

It is rarely reported in the literatures, possibly because many clinicians consider it as a normal variation and bears no clinical significance apart from being esthetically objectionable when it is extensive. (Darwazeh et al., 2012), its prevalence in this study $(7.9 \%)$, when the gender wise prevalence of MS was considered, males significantly affected $(10.2 \%)$ more than females $(5.5 \%)(P=0.047)$ and increases significantly with age $(P<0.001)$. Same as Hussein et al (2017) which diagnosed MS (16.8\%) as the most common oral condition, and Ambika et al. (2011) reported (13.16\%) for, both studies showed higher prevalence than current result, but mostly among older females, while Mumcu et al. (2005) a study among Turkish children was diagnosed MS as the most common oral condition also, but with lower prevalence $(6.9 \%)$ than current study.

It is widely known that racial pigmentations are more common in dark-skinned people; therefore, ethnic and geographic variation in its prevalence is understandable, and some factors such as smoking, hormone induced, and drug-related pigmentations are likely to increase as the age advances. Observation of different prevalence for each oral condition in different studies can be explained by various geographical areas, different cultures with different ethnic origin populations and habits, and different parameters for diagnosis might have a role.

\section{CONCLUSION AND LIMITATION}

This study found that oral conditions are common in children, some increasing with age; hence, it is the responsibility of the general dental practitioners and 
pediatric dentists to be aware of the recognition, etiology, clinical presentation, and possible association with systemic disease, as may be the earlier sign of their diagnosis hoping that it provides helpful information for further future epidemiological studies.

One should not deny weakness in this study such as doing it in a single dental center, so the results cannot represent the prevalence of actual general population. Furthermore, the diagnosis of the oral conditions was performed after only a single examination of each patient, since some need follow-up.

\section{REFERENCES}

Aldred, M.J., A. C. Cameron and R. Hall. 2008. Pediatric Oral Medicine and Pathology: Handbook of Pediatric Dentistry. $3^{\text {rd }}$ ed. Mosby Elsevier, Philadelphia, PA. p192-216.

Al-Maweri, S. A., W. A. Al-Soneidar and E. S. Halboub. 2014. Oral lesions and dental status among institutionalized orphans in Yemen: A matched case-control study. Contemp. Clin. Dent. 5(1): 81 .

Ambika, L., V. Keluskar, S. Hugar and S. Patil. 2011. Prevalence of oral mucosal lesions and variations in Indian public school children. Braz. J. Oral Sci. 10(4): 288-293.

Bessa, C. F. N., P. J. B. Santos, M. C. F. Aguiar and M. A. V. Do Carmo. 2004. Prevalence of oral mucosal alterations in children from 0 to 12 years old. J. Oral Pathol. Med. 33(1): 17-22.

Boyce, R. and J. Mull. 2008. Complying with the occupational safety and health administration: Guidelines for the dental office. Dent. Clin. North Am. 52(3): 653-668.

Cavalcante, R. B., E. Turatti, A. P. B. Daniel, G. F. de Alencar and Z. Chen. 2016. Retrospective review of oral and maxillofacial pathology in a Brazilian paediatric population. Eur. Arch. Pediatr. Dent. 17(2): 115-122.

Crespo, M., P. Del Pozo and R. García. 2005. Epidemiology of the most common oral mucosal diseases in children. Med. Oral Patol. Oral Cir. Bucal. 10: 376-87.

Darwazeh, A. M., M. M. Al-Aboosi and A. A. Bedair. 2012. Prevalence of oral mucosal lesions in psoriatic patients: A controlled study. J. Clin. Exp. Dent. 4(5): e286.

Dhanuthai, K., M. Banrai and S. Limpanaputtajak. 2007. A retrospective study of paediatric oral lesions from Thailand. Int. J. Pediatr Dent. 17(4): 248-253.

Dos Santos, P. J. B., C. F. N. Bessa, M. C. F. De Aguiar and M. A. V. Do Carmo. 2004. Cross-sectional study of oral mucosal conditions among a central Amazonian Indian community, Brazil. J. Oral Pathol. Med. 33(1): 7-12.

Furlanetto, D. L. C., A. Crighton and G. V. A. Topping. 2006. Differences in methodologies of measuring the prevalence of oral mucosal lesions in children and adolescents. Int. J. Pediatr. Dent. 16(1): 31-39.

Garcia-Pola, M. J., J. M. Garcia-Martin and M. Gonzalez-Garcia. 2002. Prevalence of oral lesions in the 6-year-old pediatric population of Oviedo (Spain). Med. Oral. 7(3): 184-191.
Ha, W. N., E. Kelloway, F. Dost and C. S. Farah. 2014. A retrospective analysis of oral and maxillofacial pathology in an Australian paediatric population. Aust. Dent. J. 59(2): 221-225.

Hussein, A. A., A. M. Darwazeh and S. H. Al-Jundi. 2017. Prevalence of oral lesions among Jordanian children. Saudi J. Oral Sci. 4(1): 12.

Jahanfar, J. D., E. M. Douglas and H. Alinejad. 2012. Prevalence of oral lesions and normal variants of the oral mucosa in 12 to 15 -yearold students in Tehran, Iran. Arch. Iran. Med. 15(3): 142-145.

Jones, A. V. and C. D. Franklin. 2006. An analysis of oral and maxillofacial pathology found in children over a 30-year period. Int. J. Pediatr. Dent. 16(1): 19-30.

Löe, H. and J. Silness. 1963. Periodontal disease in pregnancy I. Prevalence and severity. Acta Odontol. Scand. 21(6): 533-551.

Majorana, A., E. Bardellini, P. Flocchini, F. Amadori, G. Conti and G. Campus. 2010. Oral mucosal lesions in children from 0 to 12 years old: ten years' experience. Oral Surg. Oral Med. Oral Pathol. Oral Radiol. Endodontol. 110(1): e13-e18.

Mumcu, G., H. Cimilli, H. Sur, O. Hayran and T. Atalay. 2005. Prevalence and distribution of oral lesions: A cros-sectional study in Turkey. Oral Dis. 11(2): 81-87.

Parlak, A. H., S. Koybasi, T. Yavuz, N. Yesildal, H. Anul, I. Aydogan, R. Cetinkaya and A. Kavak. 2006. Prevalence of oral lesions in 13to 16-year-old students in Duzce, Turkey. Oral Dis. 12(6): 553-558.

Patil, S. S., U. R. Kontham, R. K. Kontham and A. Chowdhery. 2017. Retrospective evaluation of paediatric oral biopsies over a 10-year period in Western India. Eur. Arch. Pediatr. Dent. 18(3): 171-178.

Petersen, P. E., S. Estupinan-Day and C. Ndiaye. 2005. WHO's Action for Continuous Improvement in Oral Health. World Health Organization, Geneva.

Puangwan, L., R. Juengsomjit, P. Klanrit, S. Taweechaisupapong and S. Poomsawat. 2015. Oral and maxillofacial lesions in a Thai pediatric population: A retrospective review from two dental schools. J. Med. Assoc. Thai. 98(3): 291-297.

Sandeepa, N. C., H. P. Jaishankar, C. B. Sharath, M. S. Abhinetra, D. D. Darshan and N. Deepika. 2013. Prevalence of oral mucosal lesions among pre-university students of Kodava population in Coorg district. J. Int. Oral Health. 5(3): 35.

Shulman, J. D. 2005. Prevalence of oral mucosal lesions in children and youths in the USA. Int. J. Pediatr. Dent. 15(2): 89-97.

Stephen, J. M. 2010. Gingivitis Emedicine. Available from: http:// www.emedicine.medscape.com/article/763801-overview. [Last accessed on 2010 Oct 28].

Taweevisit, M., W. Tantidolthanes, S. Keelawat and P. S. Thorner. 2018. Paediatric oral pathology in Thailand: A 15-year retrospective review from a medical teaching hospital. Int. Dent. J. 68(4): 227-234.

Unur, M., K. B. Kayhan, M. S. Altop, Z. B. Metin and Y. Keskin. 2015. The prevalence of oral mucosal lesions in children: A single center study. J. Istanb. Univ. Facult. Dent. 49(3): 29.

Vieira-Andrade, R. G., P. A. Martins-Júnior, P. Corrêa-Faria, P. E. M. Stella, S. A. Marinho, L. S. Marques and M. L. RamosJorge. 2013. Oral mucosal conditions in preschool children of low socioeconomic status: Prevalence and determinant factors. Eur. J. Pediatr. 172(5): 675-681.

Yarom, N., U. Cantony and M. Gorsky. 2004. Prevalence of fissured tongue, geographic tongue and median rhomboid glossitis among Israeli adults of different ethnic origins. Dermatology. 209(2): 88-94. 